\title{
Optimal Speed Profile Generation for Airport Ground Movement with Consideration of Emissions
}

\author{
Jun Chen, Michal Weiszer \\ School of Engineering \\ University of Lincoln, Brayford Pool Campus \\ Lincoln, United Kingdom \\ juchen@lincoln.ac.uk, mweiszer@lincoln.ac.uk
}

\author{
Paul Stewart \\ Institute for Innovation in Sustainable Energy \\ Lonsdale House, Quaker Way \\ Derby, United Kingdom \\ p.stewart1@derby.ac.uk
}

\begin{abstract}
Emissions during the ground movement are mostly calculated based on International Civil Aviation Organisation (ICAO) emission databank. The fuel flow rate is normally assumed as a constant, hence the emission index. Therefore, no detailed discrimination of power settings during ground movement is considered to account for different emissions at different power settings. This may lead to a suboptimal and often unrealistic taxi planning. At the heart of the recently proposed Active Routing (AR) framework for airport ground movement is the unimpeded optimal speed profile generation, taking into account both time and fuel efficiency. However, emissions have not been included in the process of generating optimal speed profiles. Taking into account emissions in ground operations is not a trivial task as not all emissions can be reduced on the same path of reducing time and fuel burn. In light of this, in this paper, a detailed analysis of three main emissions at the airports, viz. CO, Total Hydrocarbon (HC), and NOx, are carried out in order to obtain a minimum number of conflicting objectives for generating optimal speed profiles. The results show that NOx has a strong linear correlation with fuel burn across all aircraft categories. For the heavy aircraft, HC and $\mathrm{CO}$ should be treated individually apart from the time and fuel burn objectives. For medium and light aircraft, a strong correlation between $\mathrm{HC}, \mathrm{CO}$ and time has been observed, indicating a reduced number of objectives will be sufficient to account for taxi time, fuel burn and emissions. The generated optimal speed profiles with consideration of different emissions will have impact on the resulted taxiing planning using the AR and also affect decisions regarding airport regulations.
\end{abstract}

Keywords-Multi-objective optimisation, Active Routing, fuel consumption, emissions, speed profile generation, airport ground movement.

\section{INTRODUCTION}

Considering the carbon emissions from a typical EU hub airport such as Heathrow, in 2014, 27\% of the total airport emissions were contributed by aircraft engines on the ground. While only a fraction of an aircraft's journey consists of the Landing and Take-Off cycle (LTO), this make an excessively large contribution to the running cost of an aircraft. This is particularly the case at the larger airports and especially for short-haul flights, as jet-engines are designed to operate optimally at cruising speed, and are considerably inefficient in the standard LTO. Moreover, a Eurocontrol report [1] has stated that there is an expected increase of $50 \%$ in flights in
2035 when compared to 2013 equating to a 1.8\% average annual increase. This is unsustainable without a change in the way that airports operate. Moreover, the EU has a clear objective to reduce CO2 by $75 \%$, NOx by $90 \%$ (per passenger and per km) in 2050 against a baseline year of 2020. It is worth noting that the level of complexity of meeting these set targets is further compounded due to the conflicting nature of $\mathrm{HC}, \mathrm{CO}$ and NOx, which are directly linked to power settings but in a completely opposite way. Furthermore, the $\mathrm{CO}$ and $\mathrm{HC}$ present a very nonlinear correlation with the low power settings. All these factors could not be appropriately addressed in a conventional single objective decision making framework. The mentioned targets will not be achievable without more effective and more efficient airport operations.

Although taxi operations are often one of the largest source of fuel burnt and emissions in a standard LTO around airports, many studies that focus on fuel consumption and emissions on the airport surface assume an average value for fuel flow during taxi, without explicitly accounting for fuel flow during idling, accelerating from a stop position, taxiing at constant speed, and turning. As a result, fuel burn, associated surface emissions, and airline's cost are thought to be reduced on the same path while reducing the taxi times. As pointed out in [2], [3], the amount of fuel consumed is an important metric for benefit assessment of congestion control methods, and its detailed estimation plays an important role in calculating the environmental impact of air traffic operations. In light of this, recently, a moving trend has been observed, employing a datadriven approach for modelling of fuel consumption and emissions [3], [4]. The aim is to distinguish contributions of different taxi phases to the total fuel consumed on the surface and its associated emissions. The conclusions drawn in [3] and [4] call for a more elaborated ground movement decision support system. Such a system should be able to address:

1) The optimal number of acceleration events: reducing the number of acceleration events will lead to less fuel consumption.

2) The optimal power setting and its duration: it is worth pointing out that assumptions made in [3] for a fixed power setting for acceleration and its duration, when applied to routing and scheduling, is not realistic and will only lead to a constrained search space, consequently 
suboptimal solutions; both of these two factors will determine collaboratively the durations spent on 'acceleration' and 'taxiing at constant speed', two largest sources of surface fuel consumption; choosing an appropriate acceleration rate and its duration to reduce the amount of times spent on these two phases will greatly reduce fuel consumption and its associated environmental impact.

As indicated in [3], there is a lack of consensus regarding what values of power settings and time required for each manoeuvre should be used. Moreover, the increase in breakaway thrust has little effect on total fuel and emission values, which implies that a slightly higher acceleration thrust may be beneficial in both time and fuel efficiency. Having a decision support system, which can take into account different acceleration thrust levels and their corresponding durations, will facilitate decision makers (DM) to evaluate the best possible practice and review regulations for a specific airport under investigation.

In [5], [6], a new conceptual framework, namely the Active Routing and Scheduling (AR), has been proposed mainly to address the mentioned trade-off between the time and fuel efficiency. Emissions are assumed to be correlated either with the time or fuel burn objectives, and are not explicitly included in optimal speed profile generation. However, due to the highly non-linear correlation between $\mathrm{HC}$ and $\mathrm{CO}$ emission index and fuel flow rate, the mentioned assumption may not hold. In light of this, in this paper, a detailed analysis of three main emissions at the airports, viz. CO, Total Hydrocarbon (HC), and NOx, are carried out in order to obtain a minimum number of conflicting objectives for generating optimal speed profiles. The results reveal a rather complex answer with regards to the formation of optimal speed profiles: for aircrafts under different weight categories, a different optimisation strategy should be adopted. The generated optimal speed profiles with consideration of different emissions will have impact on the resulted taxiing planning using the AR and also affect decisions regarding airport regulations.

The paper is organised as follows: Section II introduces the proposed multi-objective speed profile generation framework; introduction of models of aircraft motion, fuel consumption and emissions is also included in this section; computational results based on Manchester Airport are presented in Section III, where a systematic objective reduction procedure is carried out; finally, conclusions are drawn in Section IV.

\section{MULTI-OBJECTIVE OPTIMAL SPEED PROFILE GENERATION}

\section{A. Problem description}

The problem presented in this paper intends to investigate how to taxi in an efficient manner, taking into account taxi time (objective $g_{1}$ ), fuel consumption (objective $g_{2}$ ), and emissions: HC (objective $g_{3}$ ) and CO (objective $g_{4}$ ). Although usually emissions of NOx have been considered in previous research, they are not included as a separate objective here as they are linearly correlated with fuel flow. As a result, minimizing fuel consumption will minimize NOx at the same time. In order to systematically investigate different power settings affecting all these four mentioned objectives during taxiing, in the following sections, we first model the taxiing procedure as a discretized piece-wise linear speed profile. Given a speed profile, fuel consumption and emissions are estimated using the ICAO Emissions Databank [7]. The obtained decision variables, and objectives $g_{1}-g_{4}$ are then used in a multiobjective artificial immune optimisation algorithm [8] to generate optimal speed profiles.

\section{B. Discretized Piece-wise Linear Speed Profiles}

In order to model unimpeded taxiing procedure along the given route as shown in Fig. 1, the route is further divided into large segments, each containing several edges. For example, several consecutive straight edges typically form one straight segment. The turning segment consists of consecutive edges between which there is an angle of at least 30 degrees.

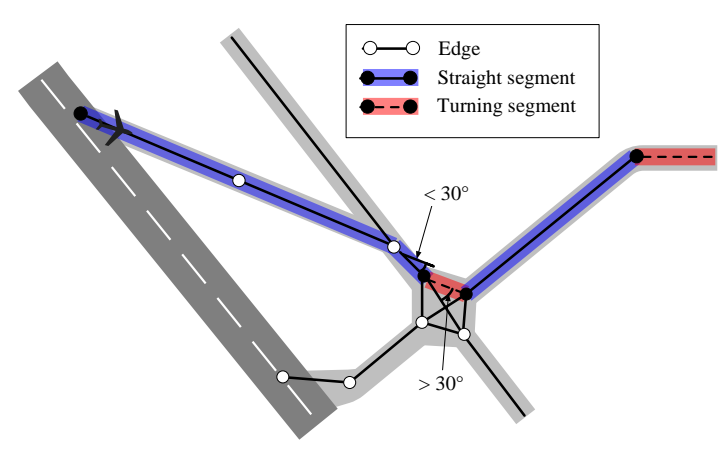

Fig. 1. An example of a taxiway at Manchester Airport, represented by segments consisting of edges.

As aircraft can taxi with speed as a continuous function of time along each segment, it gives rise to infinite degrees of freedom. In order to further reduce the complexity of the speed profile optimisation problem, each straight segment of the route is decomposed into four parts, corresponding to four different aircraft taxiing phases, i.e. acceleration, travelling at constant speed, braking and rapid braking, representing a typical taxiing behavior as illustrated in Fig. 2. Therefore, further optimisation is reduced to only find out optimal switching times of different phases.

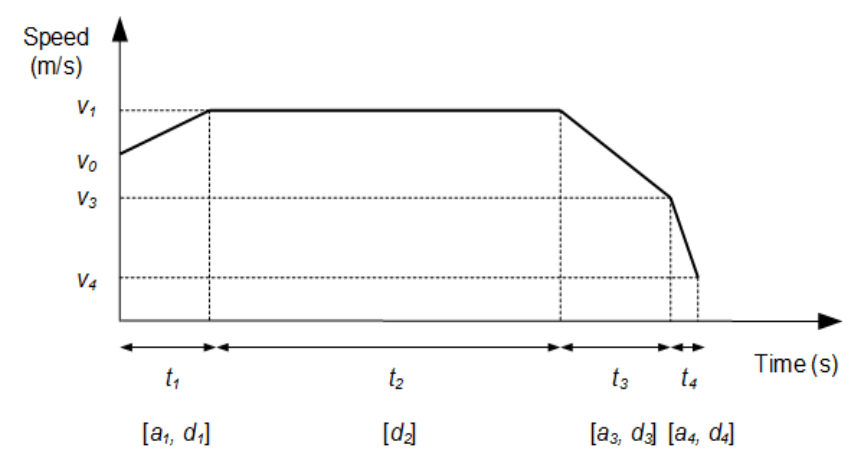

Fig. 2. An example of a speed profile with four phases. 
The first phase is the acceleration phase in which an aircraft maintains a constant acceleration rate $a_{1}$ over the distance $d_{1}$, thus increasing its speed from the initial speed $v_{0}$ at the start of the segment to $v_{1}$. During the second phase, an aircraft will traverse at the constant speed $v_{1}$ until the end of the second phase $d_{2}$ is reached. In the third and the fourth phases, an aircraft will decelerate from the speed $v_{1}$ to the speed $v_{4}$ at the end of the segment. The last two phases have different deceleration rates where, $a_{4}$ is equal to the maximum deceleration rate which enables the speed to be quickly reduced to $v_{4}$. As for the third phase, the deceleration rate $a_{3}$ will be uniquely determined by $a_{4}$ and $d_{4}$, since $v_{3}$ can be derived backwards given $a_{4}, v_{4}, d_{4}$ and the length of the third phase is equal to $d_{3}=d-d_{1}-d_{2}-d_{4}$.

For turning segments we assume that the aircraft will have a constant speed $v_{\text {turn }}$. The maximum speed on straight taxiways $v_{\text {straight }}$ is restricted to 30 knots and turning speed $v_{\text {turn }}$ is set to 10 knots as in [9]. The consecutive segments are linked together so that the final speed $v_{4}$ of the preceding segment is the initial speed $v_{0}$ of the subsequent segment. Furthermore, the maximum acceleration and deceleration rate $a_{\text {max }}$ is set to $0.98 \mathrm{~m} \cdot \mathrm{s}^{-2}$ for passenger comfort, similar as in [10]. As a result, there are four independent variables $a_{1}, d_{1}, d_{2}, d_{4}$ which define a unique speed profile over a segment. By searching for values of these four variables, one can explore different speed profiles with different taxi time, fuel consumption, and emissions.

The taxi time $\left(g_{1}\right)$ needed to traverse a single segment is the sum of the time $t_{j}$ spent in the different phases.

$$
g_{1}=\sum_{j=1}^{4} t_{j}
$$

\section{Fuel Consumption Calculation}

In order to calculate fuel consumption $\left(g_{2}\right)$ of the participating aircraft, its longitudinal motion model is derived in (2) by considering the following forces: thrust $T$ generated by the engines, normal force $m \cdot g$, where $g=9.81 \mathrm{~m} \cdot \mathrm{s}^{-2}$ and rolling resistance of tyres $F_{r}$. As the speed during taxiing is rather low, the aerodynamic drag is not considered here.

$$
T=m \cdot a_{1}+F_{r}
$$

Where, $F_{r}$ is proportional to the rolling resistance coefficient $\mu$ and normal force $m \cdot g$ as given in (3). The coefficient $\mu$ is suggested to be around 0.02 for aircraft tyres [11]. In this paper, $\mu$ is set to 0.015 for concrete surface.

$$
F_{r}=\mu \cdot m \cdot g
$$

Given a particular speed profile, the associated thrust $T$ is defined by (2). Given $T$ and maximum power output $F_{0}$ of the engine, the thrust level $\varepsilon$ can be calculated:

$$
\varepsilon=\frac{T}{F_{0}} .
$$

As mentioned in Section III.A, four phases are defined for a straight segment: acceleration, constant speed, braking and rapid braking. Equation (4) is used for acceleration and constant speed phase. During braking and rapid braking, we assume $\varepsilon=5 \%$. For turning, $\varepsilon=7 \%$. The fuel flow $f$ corresponding to the thrust level $\varepsilon$ is obtained by linear interpolation/extrapolation from ICAO Emissions Databank at $7 \%$ and $30 \%$ similarly as in [3]. Finally, the fuel consumption $\left(g_{2}\right)$ for the segment is obtained by multiplication of fuel flow $f_{j}$ for the specific phase $j$ and the time $t_{j}$ spent in this state:

$$
g_{2}=\sum_{j=1}^{4} f_{j} \cdot t_{j}
$$

\section{Emissions estimation}

The ICAO Emissions Databank used for fuel consumption calculation lists emissions data for a majority of commercial engines. For each engine type, it provides values of fuel flow $(\mathrm{kg} / \mathrm{s})$ and emission indices (EI) (g of pollutant emitted per $\mathrm{kg}$ of fuel burnt) taken at $7 \%, 30 \%, 85 \%$ and $100 \%$ rated outputs as shown in Fig. 3. The pollutants included in the Databank are $\mathrm{HC}, \mathrm{CO}$, and NOx. Using reported values of EIs taken at 7\%, $30 \%, 85 \%$ and $100 \%$, a curve can be fitted to model the relationship between EI and fuel flow. Specifically, HC and CO is modelled as exponential function whereas NOx is fitted with a linear function. Given a calculated fuel flow as described in Section II.C, the corresponding $E I_{j}^{p}$ for pollutant $p$ during phase $j$ can be obtained using the fitted curves. Then, the total value of emission emitted when traversing the segment is calculated as given in (6).

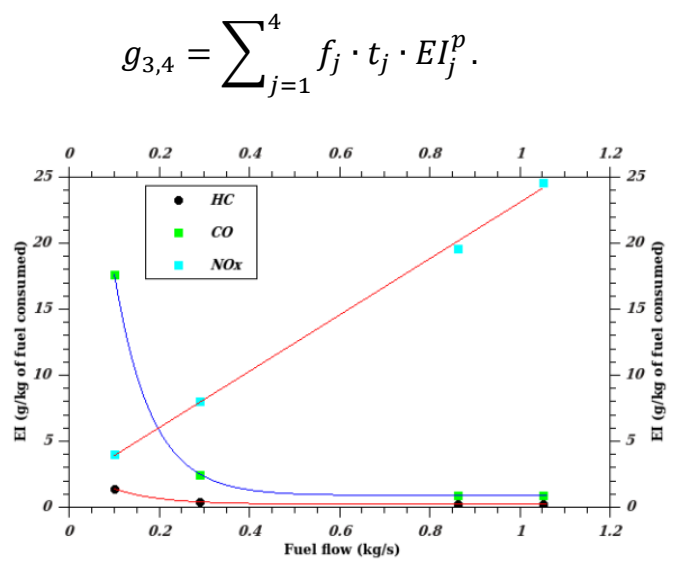

Fig. 3. Emission indices as a function of fuel flow for a CFM56-5-A1 jet engine. Note: The four points on each trend line correspond to levels of engine thrust $7 \%, 30 \%, 85 \%$ and $100 \%$, from left to right.

\section{E. A Population Adaptive Algorithm}

To solve the speed profile optimisation problem introduced in Section II.A, the Population Adaptive Immune Algorithm 
(PAIA) [8] is used to investigate different combinations of decision variables $a_{1}, d_{1}, d_{2}, d_{4}$. PAIA is briefly outlined in Fig 4.

\begin{tabular}{|ll|}
\hline 1: & Generate the fastest speed profile for all segments; \\
2: & Generate initial population around this solution; \\
3: & Identify non-dominated solutions; \\
4: & For gen = 1 to gen $n_{\max }$ do: \\
5: & $\quad \begin{array}{l}\text { Calculate the fitness of the solutions based on } \\
\text { the distance from the reference solution; } \\
\text { Select good solutions according to their fitness } \\
\text { value and clone them; } \\
\text { Mutate the Cloned solutions; } \\
\text { 7: }\end{array} \quad \begin{array}{l}\text { Check mutated clones for constraints } \\
\text { violations; } \\
\text { Combine solutions, evaluate them and identify } \\
\text { non-dominated ones; } \\
\text { Re-select solutions which will be passed to the } \\
\text { 10: }\end{array}$ \\
11: & End
\end{tabular}

Fig. 4. PAIA for optimal speed profile generation.

In order to speed up the search, a solution with the shortest taxi time for the given route is first analytically derived in line 1. The generated solution is then seeded into the initial population. The rest of the initial population is filled by solutions randomly generated around the seeded solution (line 2). Solutions are evaluated in line 3 in terms of the taxi time and fuel consumption and non-dominated sorting is performed in order to identify non-dominated solutions. The algorithm iterates for gen $_{\max }$ iterations (lines 4-11). In line 5, one of the non-dominated solutions is randomly selected to be a reference solution for fitness calculation. Each solution is assigned a corresponding fitness which is the distance from the reference solution. The solutions are then selected for cloning based on their fitness values (line 6). The solutions with a good fitness value are cloned into more clones than worse solutions. The fitness value of solutions also affects their mutation in line 7. Good solutions undergo only small variation whereas worse solutions are mutated with a higher degree in order to explore new areas of the search space. Next, the mutated clones are checked for feasibility and constraints violations (line 8). The clones are combined with the previous solutions and evaluated (line 9). The non-dominated solutions are always selected to survive into the next generation and only if their number is less than the size of the initial population, solutions from the next fronts are chosen based on their fitness value. The output of the algorithm is an approximation of the Pareto front. For detailed implementation and constraint handling, readers are referred to [5], [12].

\section{COMPUTATIONAL RESUlTS}

The algorithm was tested on a set of routes on Manchester Airport, the largest airport in United Kingdom outside London with 2 runways. Between each of 98 gate/stands and 4 runway exits for both departing and arriving aircraft, the shortest route are generated. In total, $98 \cdot 4 \cdot 2=784$ routes are created. Then, for each route the speed profile optimisation problem was solved for each representative aircraft, defined as follows.
Three representative aircraft were designated according their wake vortex separation requirements (light, medium, heavy). Specifications of the representative aircraft were used during the calculation. The specifications are summarized in Table I. The algorithm was implemented in Matlab ${ }^{\circledR}$ programming language. Pareto fronts of trade-off solutions yielded by solving the speed profile optimisation problem for 3 representative aircraft taxiing on the generated routes are examined in the following sections.

TABLE I. SPECIFICATIONS OF THE RESPRESENTATIVE AIRCRAFT

\begin{tabular}{lccc}
\hline \hline \multicolumn{1}{c}{ Weight category } & light & medium & heavy \\
\hline \hline Representative aircraft & Learjet 35A & Airbus A320 & Airbus A333 \\
Take-off weight $m$ & $8300 \mathrm{~kg}$ & $78000 \mathrm{~kg}$ & $230000 \mathrm{~kg}$ \\
Engines & TFE731-2-2B & CMF56-5-A1 & CF6-80E1A2 \\
Number of engines & 2 & 2 & 2 \\
Rated output $F_{o}$ & $2 \times 15.6 \mathrm{kN}$ & $2 \times 111.2 \mathrm{kN}$ & $2 \times 287 \mathrm{kN}$ \\
Rolling resistance $F_{r}$ & $1221 \mathrm{~N}$ & $11.48 \mathrm{kN}$ & $33.84 \mathrm{kN}$ \\
Fuel flow at $7 \% F_{o}$ & $0.024 \mathrm{~kg} . \mathrm{s}^{-1}$ & $0.101 \mathrm{~kg} . \mathrm{s}^{-1}$ & $0.228 \mathrm{~kg} . \mathrm{s}^{-1}$ \\
Fuel flow at $30 \% F_{o}$ & $0.067 \mathrm{~kg} . \mathrm{s}^{-1}$ & $0.291 \mathrm{~kg} . \mathrm{s}^{-1}$ & $0.724 \mathrm{~kg} . \mathrm{s}^{-1}$ \\
\hline \hline
\end{tabular}

\section{A. Visual comparison of Pareto fronts}

By visually comparing the Pareto fronts of optimal solutions, the following conclusions can be made. Firstly, as shown in Fig. 5 for a light category aircraft, there is an apparent relation between $\mathrm{HC}$, $\mathrm{CO}$ on one hand and taxi time or fuel consumption on the other. With lower fuel consumption values, $\mathrm{HC}$ and $\mathrm{CO}$ tend to be high and vice versa. Similarly, solutions with a short taxi time generate low emissions. Secondly, the obtained optimal solutions show a limited tradeoff in terms of emissions, this also holds for a medium category aircraft. On the other hand, Pareto front for a heavy category aircraft present a trade-off between different objectives, as shown in Fig. 6. The results suggest that the speed profiles with less taxi time and hence large acceleration yield lower emissions than the fuel efficient speed profiles with lower acceleration.

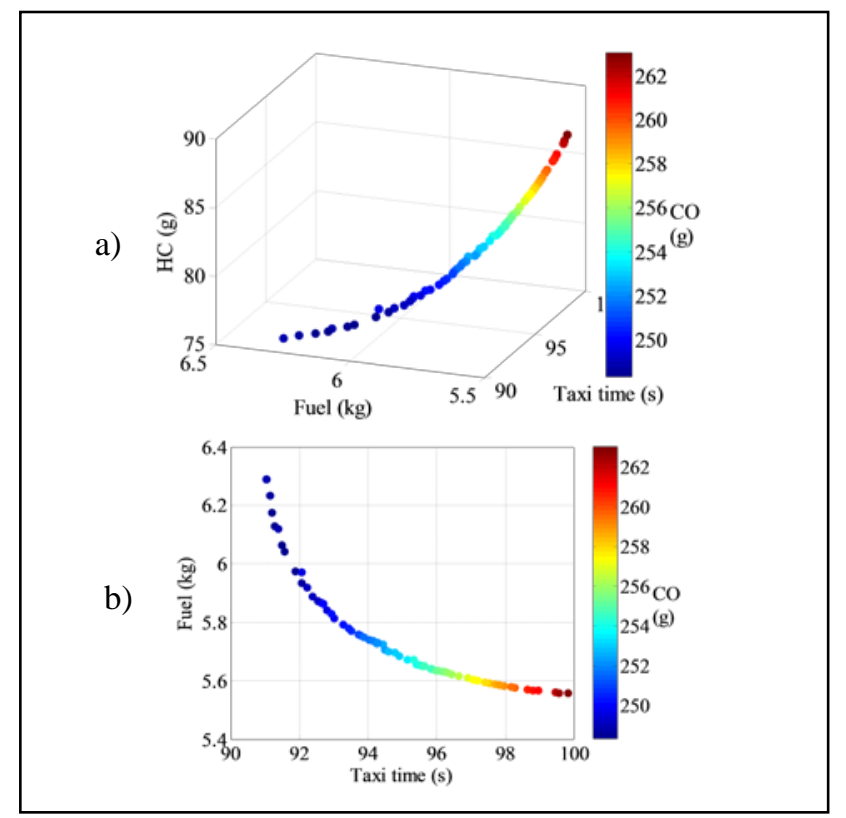

Fig. 5. Pareto front for a light category aircraft, projected to $g_{1}-g_{3}$ (a) and $g_{1}-g_{2}$ (b). 


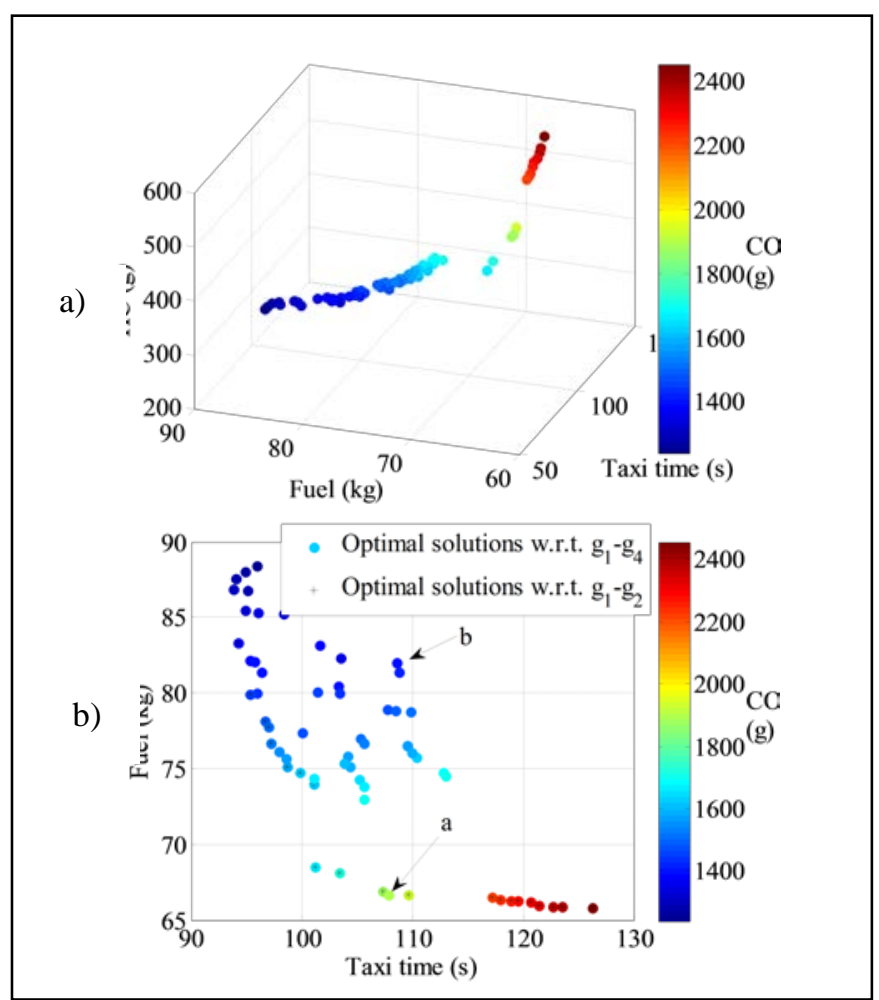

Fig. 6. Pareto front for a heavy category aircraft, projected to $g_{1}-g_{3}$ (a) and $g_{1}-g_{2}$ (b).

In order to analyse the obtained optimal speed profiles, the solutions $a, b$ from Fig. $6 \mathrm{~b}$ are shown in Fig. 7 and their objective values are given in Table III. As can be seen in Fig. 7 , the two speed profiles differ considerably, notably in the acceleration phase. Longer acceleration in case of solution $b$ resulted in higher fuel flow and hence fuel consumption, however, higher fuel flow also caused that $\mathrm{HC}$ and $\mathrm{CO}$ emissions are lower as emissions decrease with higher fuel flow values as shown in Fig. 3.

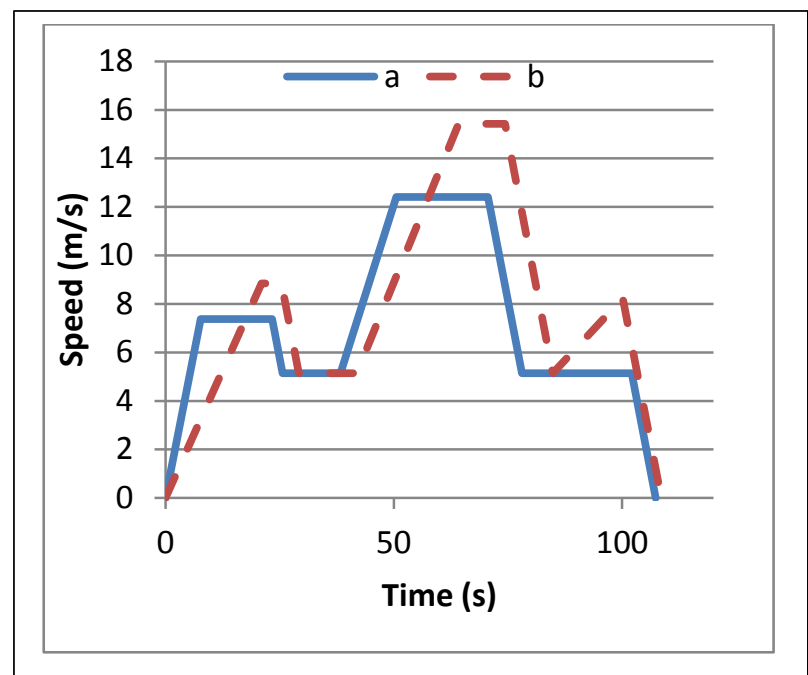

Fig. 7. Optimal speed profiles given by solutions (a) and (b).
TABLE II. DIFFERENCE BETWEEN SOLUTIONS

\begin{tabular}{ccc}
\hline & Solution $a$ & Solution $b$ \\
Taxi time (s) & 107.40 & 108.60 \\
Fuel (kg) & 66.87 & 81.98 \\
HC (g) & 448.50 & 292.50 \\
CO (g) & 1882.10 & 1401.80 \\
\hline
\end{tabular}

The relationship between objectives is further examined in correlation analysis in the next section.

\section{B. Correlation between Objectives}

The obtained correlation matrix is shown in Table III. As can be observed from this matrix, taxi time and fuel consumption are negatively correlated, i.e., they are in conflict with each other, as noted in the previous research [3]. On the other hand, both $\mathrm{HC}$ and $\mathrm{CO}$ emissions are positively correlated with taxi time and negatively correlated with fuel. Furthermore, the values of correlation for $\mathrm{HC}$ and $\mathrm{CO}$ are very similar, proving the same behaviour of objectives $g_{3}, g_{4}$ as both pollutants are exponentially related to fuel flow. Secondly, all three objectives: taxi time, HC and CO are strongly correlated and non-conflicting. However, each of them is in conflict with the fuel consumption. Thus, this result indicates that HC and CO objectives are redundant in this problem, since they are correlated to taxi time. In the next section, we investigate if it is possible to omit $\mathrm{HC}$ and $\mathrm{CO}$ from the optimisation problem and how does it affect the DM in selecting solutions.

TABLE III. CORRELATION BETWEEN OBJECTIVES

\begin{tabular}{crrrr}
\hline \hline \multicolumn{5}{c}{ Light } \\
\hline & $\begin{array}{c}\text { Taxi time } \\
\text { (s) }\end{array}$ & Fuel (kg) & HC (g) & CO (g) \\
Taxi time (s) & 1 & -0.8797 & 0.9939 & 0.9891 \\
Fuel (kg) & -0.8797 & 1 & -0.9143 & -0.8200 \\
HC (g) & 0.9939 & -0.9143 & 1 & 0.9783 \\
CO (g) & 0.9891 & -0.8200 & 0.9783 & 1 \\
\hline \multicolumn{5}{c}{ Medium } \\
\hline Taxi time (s) & 1 & -0.8759 & 0.9954 & 0.9822 \\
Fuel (kg) & -0.8759 & 1 & -0.9004 & -0.9365 \\
HC (g) & 0.9954 & -0.9004 & 1 & 0.9871 \\
CO (g) & 0.9822 & -0.9365 & 0.9871 & 1 \\
\hline Taxi time (s) & 1 & -0.814 & 0.8547 & 0.8972 \\
Fuel (kg) & -0.814 & 1 & -0.931 & -0.9407 \\
HC (g) & 0.8547 & -0.931 & 1 & 0.9914 \\
CO (g) & 0.8972 & -0.9407 & 0.9914 & 1 \\
\hline \hline
\end{tabular}

\section{Objective reduction}

The results in the previous section indicate that some of the objectives, namely HC and CO may be redundant in the speed profile optimisation problem and it may be sufficient to perform optimisation with regard to taxi time and fuel consumption only. However, the question arises if we omit some of the objectives, how the obtained optimal solutions would differ from the 'truly' optimal solutions w.r.t. to the original set of objectives.

Suppose that Fig. 6 shows all Pareto optimal solutions (speed profiles) $x \in X$ for the given aircraft and route, w.r.t to 
objective functions $g_{1}-g_{4}$. The fact they are Pareto optimal means they are incomparable, i.e. no solution is better than other in all objectives $g_{1}-g_{4}$. However, by considering only a subset of objectives, e.g. $g_{1}, g_{2}$, some solutions become comparable. For example, solutions $a$ and $b$ in Fig. 6 are incomparable as can be seen in Table III. Now, if we assume that only $g_{1}$ and $g_{2}$ are considered, as a result, solution $a$ will dominate solution $b$, since $a$ is better in $g_{1}$ and $g_{2}$. Although, in reality, $a$ does not dominate $b$ w.r.t. $g_{1}-g_{4}$, as $g_{3}(a)>g_{3}(b)$ and $g_{4}(a)>g_{4}(b)$. Formally, the difference between solutions obtained by optimisation w.r.t. to all objectives in set $F$ and solutions obtained by optimisation with omitting objective $g_{k}$, that is w.r.t. $F-\left\{g_{k}\right\}$, can be defined as $\delta_{k}$ error measure [13]. Whenever a solution $x$ dominates solution $y$ w.r.t. $F-\left\{g_{k}\right\}$ the difference can be calculated as given in (7).

$$
\delta_{k}=g_{k}(x)-g_{k}(y) .
$$

In other words, $\delta_{k}$ specifies the additive term that has to be added to objective values of the obtained solutions such that the original dominance structure is maintained. In the given example case, by assuming that $a$ dominates $b$, and subsequently choosing $a$ as a solution that is going to be implemented, we make error $\delta_{3}=448.50-292.50=156 \mathrm{~g}$ and $\delta_{4}=1882.10-1401.80=480.3 \mathrm{~g}$. Then, by computing the $\delta_{k}$ values for all solution pairs $x, y \in X$ of all aircraft and all routes, we can then determine the maximum error. The meaning of the maximum $\delta_{k}$ value is that whenever the DM wrongly assumes that $x$ weakly dominates $y$ w.r.t. an objective subset $F$ - $\left\{g_{k}\right\}$, we also know that $x$ is not worse than $y$ in $g_{k}$ by an additive term of $\delta_{k}$. To facilitate the comparison between different aircraft and routes, we calculate $\delta_{k}$ as a ratio to the objective value range for the given aircraft:

$$
\delta_{k}^{\%}=\frac{g_{k}(x)-g_{k}(y)}{\max \left(g_{k}(x)\right)-\min \left(g_{k}(y)\right)}, x, y \in X
$$

The values of maximum and mean $\delta_{3,4}{ }^{\%}$ for all representative aircraft and all generated routes are given in Table IV. As can be seen from the table, maximum and mean delta error for medium and light category aircraft are generally low, indicating that omitting $g_{3}$ or $g_{4}$ has little effect on the optimization results. Therefore, by performing optimisation considering only $g_{1}$ and $g_{2}$ objective and then calculating $g_{3}$ and $g_{4}$ for resulting solutions is sufficiently accurate for the DM. However, for the heavy category aircraft higher values of maximum and mean $\delta$ error show that there is a clear trade-off between $g_{1}-g_{4}$. In this case, omitting $g_{3}$ or $g_{4}$ would result in bigger error, which in one case can be up to $54.61 \%$.

\section{CONCLUSIONS}

In this paper, a detailed analysis of three main emissions at
TABLE IV. DELTA ERROR

\begin{tabular}{ccccccc} 
Weight cat. & \multicolumn{2}{c}{ heavy } & \multicolumn{2}{c}{ medium } & \multicolumn{2}{c}{ light } \\
\hline & $\operatorname{Max} \delta$ & $\operatorname{Mean} \delta$ & $\operatorname{Max} \delta$ & $\operatorname{Mean} \delta$ & $\operatorname{Max} \delta$ & Mean $\delta$ \\
HC \% & $54.61 \%$ & $17.13 \%$ & $1.27 \%$ & $0.42 \%$ & $3.82 \%$ & $2.02 \%$ \\
CO \% & $35.35 \%$ & $10.56 \%$ & $5.69 \%$ & $2.88 \%$ & $1.76 \%$ & $0.95 \%$
\end{tabular}

the airports (CO, HC, Nox), are carried out in order to obtain a minimum number of conflicting objectives for generating optimal speed profiles. The results show that NOx has a strong linear correlation with fuel burn across all aircraft categories. For the heavy aircraft, $\mathrm{HC}$ and $\mathrm{CO}$ should be treated individually apart from the time and fuel burn objectives. For medium and light aircraft, a strong correlation between HC, $\mathrm{CO}$ and time has been observed, indicating a reduced number of objectives will be sufficient to account for taxi time, fuel burn and emissions. The generated optimal speed profiles with consideration of different emissions will have impact on the resulted taxiing planning using the $\mathrm{AR}$ and also affect decisions regarding airport regulations. Furthermore, as the generated speed profiles consider taxiway configurations, the optimal airport layout could be investigated in the future.

\section{REFERENCES}

[1] Eurocontrol, Challenges of Growth 2013: European Air Traffic in 2035, Tech. rep., Eurocontrol (2013).

[2] G. B. Chatterji, Fuel Burn Estimation using Real Track Data, $11^{\text {th }}$ AIAA Aviation Technology, Integration, and Operations (ATIO) Conf., 2011.

[3] T. Nikoleris, G. Gupta, M. Kistler, Detailed estimation of fuel consumption and emissions during aircraft taxi operations at Dallas/Fort Worth International Airport, Transportation Research Part D: Transport and Environment, vol. 16, Issue 4 (2011), pp. 302-308.

[4] H. Khadikar, H. Balakrishnan, Estimation of Aircraft Taxi Fuel Burn using Flight Data Recorder Archives, Transportation Research Part D: Transport and Environment, vol. 17, Issue 7 (2012), pp. 532-537.

[5] J. Chen, M. Weiszer, P. Stewart and M. Shabani, Towards A More Cost Effective and Environmental Friendly Airport Surface Movement through Active Routing Part 1: Optimal Speed Profile Generation, IEEE Trans. Intell. Transp. Syst. (2015 submitted).

[6] J. Chen et al., Towards a More Cost Effective and Environmental Friendly Airport Surface Movement through Active Routing Part 2: A Multi-objective Optimisation Approach, IEEE Trans. Intell. Transp. Syst. (2015 submitted).

[7] ICAO Aircraft Engine Emissions Databank, EASA. (2014).

[8] J. Chen and M. Mahfouf, "Population Adaptive Based Immune Algorithm for Solving Multi-objective Optimisation Problems,” in Proc. ICARIS 2006, LNCS, vol. 4163, 2006, pp. 280-290.

[9] A-SMGCS Manual, ICAO, 2004.

[10] D. L Bakowski et al., “NextGen Flight Deck Surface Trajectory-Based Operations (STBO): Speed-Based Taxi Clearances,” in Proc. Int. Symp.. Aviation Psychology, Dayton, OH, 2011, pp. 44-49.

[11] D. J. Mitchell, "Calculation of ground performance in take-off and landing”, ESDU, London, UK, Data Sheet 85029, 1985.

[12] J. Chen and P. Stewart, "Planning aircraft taxiing trajectories via a multi-objective immune optimisation," in Proc. Seventh International Conference on Natural Computation (ICNC), 2011, pp. 2235-2240.

[13] D. Brockhoff, and E. Zitzler. "Objective reduction in evolutionary multiobjective optimization: theory and applications." Evolutionary

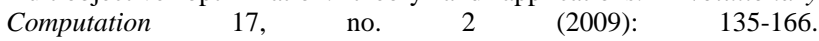

